\title{
Three Dimensional Evaluation of Marginal Microleakage at the Adhesive Interface between Different Fissure Sealants and Enamel: Micro-CT Analysis
}

\begin{abstract}
SUMMARY
Background/Aim: Sealing ability and adhesion to enamel of fissure sealants are quite critical in prevention of dental caries. The objective of the present study was to assess the marginal microleakage of different material-based pit and fissures with non-invasive and invasive techniques by using the Micro-CT method. Material and Methods: 30 extracted human permanent molars were randomly assigned to three sealant groups $(n=10)$ as resin-based ClinPro, giomer-based BeautiSealant, and glass ionomer-based Fuji IX-GP. Half of each group was randomly divided into two parts as non-invasive and invasive techniques. The volumetric analysis of marginal microleakage between the enamel-material interface was performed by using Micro-CT. The results were analyzed with Student's $t$, One-Way ANOVA, Tukey and Games-Howell tests. Statistical significance level was considered as 0.05. Results: In non-invasive technique, Fuji $I X-G P$ showed statistically significantly lower marginal microleakage values than Clinpro $(p=0.022)$. Other comparisons were not significant $(p>0.05)$. However, in invasive technique, microleakage values both of Fuji $I X-G P$ and BeautiSealant were significantly lower than Clinpro $(p<0.001$, $p<0.001)$. Besides, regardless of the sealant material, the invasive technique showed significantly superior results than non-invasive technique regarding marginal microleakage. Conclusions: Considering the clinical conditions, it was concluded that glass ionomer-based Fuji IX-GP and giomerbased BeautiSealant could be used as alternative to resin based sealants. Additionally, invasive technique (fissure preparation) can be recommended to reduce marginal microleakage.
\end{abstract}

Key Words: Micro-Computed Tomography, Microleakage, Initial Caries, Fissure Sealant, Caries Prevention

\author{
Akif Demirel ${ }^{1}$, Ayșe Ișıl Orhan ${ }^{2}$, \\ Firdevs Tulga Öz ${ }^{1}$, Kaan Orhan ${ }^{3}$ \\ ${ }^{1}$ Pediatric Dentistry, Faculty of Dentistry, \\ Ankara University, Ankara, Turkey \\ ${ }^{2}$ Pediatric Dentistry, Faculty of Dentistry, \\ Yıldırım Beyazıt University, Ankara, Turkey \\ ${ }^{3}$ Department of Dentomaxillofacial Radiology, \\ Faculty of Dentistry and Ankara University \\ Medical Design Application and Research Center \\ (MEDITAM), Ankara University, Ankara, Turkey
}

\section{ORIGINAL PAPER (OP)}

Balk J Dent Med, 2022;7-14

\section{Introduction}

Fissure caries in children is the most common type of caries caused by deep pits and fissures ${ }^{1,2}$. The deeper pits and fissures on the occlusal surfaces has become a risk factor for the development of dental caries due to unfavorable morphology and difficulties in cleaning. Also, deep grooves on pits and fissures are areas for the accumulation of debris, plaque, and microorganisms. Also, complex morphology makes pits and fissures inconvenient for access to saliva compounds and reduces the preventive effects of fluoride applications ${ }^{1,2}$.

In recent years, the concept of minimally invasive dentistry, which is more conservative in caries management and treatment approaches, has been developed and the use of caries-preventive materials 
has increased $^{3}$. Pit and fissure sealants, one of these preventive applications, prevent or slow down the development of dental caries by acting as a barrier between the deep occlusal grooves and the oral flora, food debris, and acid-producing microorganisms. Sealants are the most appropriate preventive method for non-cavitated caries lesions involving pits and fissures on the tooth surfaces. Besides, the fluoride-releasing of some fissure sealants potentiates their anti-carious effects ${ }^{2,4,5}$.

Pit and fissure sealants differ in their content ${ }^{5}$. In preventive dentistry, although the sealants are generally resin or glass ionomer based, there are recent clinical uses of glass carbomer, ormoser, and giomer based materials ${ }^{5,6}$. Fissure sealants also differ regarding the methods of application. Sealants can be placed by using non-invasive or invasive (surface preparation) technique ${ }^{7,8}$. In the invasive technique, the sealant material is applied after the widening and deepening of the pits and fissures with varying instruments/methods. This technique allows cleaning the depth of fissures, managing incipient caries, and determining the degree of spread of caries lesion towards the dento-enamel junction. It is also stated that the invasive technique increases the sealing ability, the retention, and adaptation of the sealant material ${ }^{7-10}$. Microleakage at the sealant-tooth interface is a significant criterion in predicting the clinical retention of the material $^{11}$. Microleakage which is observed between tooth surfaces and dental materials causes bacterial invasion, loss of sealant material, secondary caries as a result of the progression of the incipient caries lesion ${ }^{7,11,12}$.

To date, methods such as dye penetration, radioisotope method, acetate peel technique, confocal laser scanning, and micro computed tomography (Micro$\mathrm{CT}$ ) have been used to evaluate the microleakage in dentistry ${ }^{13}$. Recently, Micro-CT, non-destructive method, provides the advantage of obtaining continuous images, which allows determination of the deepest point of the marginal microleakage ${ }^{12,14}$. Three-dimensional reconstruction of the object to be examined in micro-CT imaging can be evaluated by slicing in any direction to obtain more accurate information ${ }^{14}$. In the literature, not many studies have evaluated the microleakage of fissure sealants with non-invasive and invasive techniques.

The aim of this study was to evaluate and compare the marginal microleakage of different pit and fissure sealants including ClinPro, BeautiSealant, and Fuji IX applied with non-invasive and invasive technique.

\section{Material and Methods}

\section{Ethical Approval}

This study was conducted in accordance with Declaration of Helsinki and CRIS guidelines for in-vitro studies ${ }^{15}$. The study procedures was approved by the
Ethics Committee of Ankara University Faculty of Dentistry (Approval Number: No:06/06; Decision Date:17.06.2020). Additionally, the written informed consent forms about the study protocol were signed by the parents of the child patients whose previously extracted teeth were used in the study.

\section{Sample Size Calculation and Including Criteria}

Recently extracted 30 permanent molar teeth were included in this study according to some inclusion criteria. Mostly, molar teeth extracted due to orthodontic reasons, controlled balance and compensation extractions or tooth impaction were used. The inclusion criteria for permanent molars were in the following: i) molar teeth with deep pits and fissures, ii) with no visible occlusal caries lesion, iii) previously non-sealed or non-restored. The extracted molars had been stored in a $1 \%$ thymol solution until the study procedures. The teeth were kept in $2.5 \%$ sodium hypochlorite for $48 \mathrm{~h}$ to effectively remove organic debris, food, tissue, and blood remnants and the teeth were cleaned by brushing with a low speed rotating brush under water-cooling. The extracted teeth were then controlled under a stereomicroscope to detect the presence of developmental defects, clefts, or cracks. In the case of the presence of these conditions, the teeth were replaced with samples that meet the mentioned criteria. Afterward, the samples were randomly assigned to three study groups.

\section{Description of the Study Groups and Treatment Procedures}

The pit and fissure sealants were applied to half of the samples in each group using the non-invasive technique and to the other half using the invasive technique, provided that it was randomized. In invasive technique, pits and fissures were widened (in a $0.3 \mathrm{~mm}$ dimension) with a tapered fissure diamond bur and a lowspeed handpiece (micromotor) under water spray. For the study samples subjected to non-invasive technique, no surface preparation of the pits and fissures were performed.

Group 1- Resin-based fissure sealant- Clinpro (3M ESPE, St Paul, USA): After the teeth were prepared for the sealant application, the Clinpro sealant was applied. The pits and fissures of the teeth were etched for $20 \mathrm{sec}$ with 37\% phosphoric acid (ETCH-37 TM, Bisco Inc., USA), then gently washed and dried with oil- and waterfree air spray. The sealant material was placed using the syringe needle tip to the pits and fissures and was manipulated with a dental explorer for avoiding possible air bubbles. Then, it was cured with light for $10 \mathrm{sec}$ with a curing light (Freelight 2 Elipar TM, 3M ESPE, Ireland).

Group 2- Giomer based fissure sealantBeautiSealant (Shofu Dental, Kyoto, Japan): After the teeth were prepared for the sealant application, BeautiSealant was applied. The primer (Beautiprimer, 
Shofu Dental, Kyoto, Japan) was applied to the pits and fissures of the teeth for $5 \mathrm{sec}$ with a micro-brush and then dried for $5 \mathrm{sec}$ with oil- and water-free air spray. The sealant material was placed using the syringe needle tip to the pits and fissures and was manipulated with a dental explorer for avoiding possible air bubbles. Then, it was cured with light for $10 \mathrm{sec}$ with a curing light (Freelight 2 Elipar TM, 3M ESPE, Ireland).

Group 3- Glass ionomer based fissure sealant- Fuji IX GP (GC Corp, Tokyo, Japan): After the teeth were prepared for the sealant application, Fuji IX GP was applied. First, the GC cavity conditioner was applied to the pits and fissures of the teeth for $10 \mathrm{sec}$ using a cotton pellet, then washed with water and dried with oiland water-free air spray. The powder and liquid of the material (powder/liquid ratio: $3.6 \mathrm{~g} / 1.0 \mathrm{~g}$ ) were mixed within $25 \mathrm{sec}$. The mixed sealant material was placed to the pits and fissures using a suitable hand instrument and was condensed and shaped with a small round-end hand instrument for avoiding possible air bubbles. When the setting was obtained, the sealant surface was coated with light-cured Fuji Coat ${ }^{\mathrm{TM}}$ LC. Then, it was cured with light for $10 \mathrm{sec}$ with a curing light (Freelight 2 Elipar TM, 3M ESPE, Ireland). Finishing procedures were performed under water cooling.

\section{Sample Preparation for Micro-CT Analysis}

The teeth sealed with different pit and fissure sealants were stored in distiled water at $24{ }^{\circ} \mathrm{C}$ for 48 $\mathrm{h}$ before Micro-CT analysis. The samples were then thermocycled to reflect intraoral thermal changes for 5000 cycles in water at 5 and $55{ }^{\circ} \mathrm{C}$. The roots and the other tooth surfaces were coated with nail varnish approximately $1 \mathrm{~mm}$ from the pit and fissure sealant. The crowns of the teeth were kept in 50\% silver nitrate $\left(\mathrm{AgNO}_{3}\right)$ for $12 \mathrm{~h}$ in an unlit room. After then, all the samples were rinsed under tap water for $5 \mathrm{~min}$ and immersed in photo-developing solution for $8 \mathrm{~h}$.

\section{Micro-CT Evaluation}

For Micro-CT evaluation of the presented study, a micro-CT device (Bruker Skyscan 1275, Kontich,
Belgium) in high resolution was used for scanning the tooth samples. Scanning properties were: $100 \mathrm{kVp}$, 100-mA beam current, $0.5-\mathrm{mm} \mathrm{Al} / \mathrm{Cu}$ filter, $10.1 \mu \mathrm{m}$ pixel size, rotation at 0.5 step. The detector was aircalibrated prior each scanning to decrease ring artifacts. Samples were rotated $360^{\circ}$ for the integration for $5 \mathrm{~min}$. The mean time of scanning was almost $120 \mathrm{~min}$. The beam hardening correction was set to $40 \%$ according to instructions of the manufacturer. Further more, the input of optimal contrast limits $\left(0-5 \times 10^{-4}\right)$ based on prior scanning and reconstruction of the specimens.

\section{Micro-CT Analysis}

The NRecon (v.1.7.10.4, SkyScan, Kontich, Belgium) and CtAn (v1.18.1.2, SkyScan) softwares were used for the quantitative measurements and the visualization of the samples, which used the modified algorithms described by Feldkamp et al. ${ }^{16}$ to obtain axial, two-dimensional, $1000 \times 1000$ pixel images. By using the NRecon software, images obtained by the scanner were reconstructed to show two-dimensional slices. In total app. 1024 cross-sectional images were reconstructed from whole volume. Moreover, The CTAn software was used for the three-dimensional volumetric visualization, analysis, and volumetric measurement. The reconstructed images were also further processed in Skyscan CTVox (v.3.3.0, SkyScan, Kontich, Belgium) for visualization. All reconstructions were made with a TFT medical display (0.3 inch flat-panel color-active matrix) (NEC MultiSync MD215MG-Munich-Germany) in 2048-2560 resolution at $75 \mathrm{~Hz}$ and $0.17-\mathrm{mm}$ dot pitch operated at 11.9 bits.

Afterthat, a volume of interest (VOI) was drawn to include entire enamel within the sample using CTAn, in which all specifications of the program were used to calculate $\mathrm{AgNO}_{3}$ penetration volume under the fissure sealant. Grayscale thresholds were defined to separate enamel from fissure sealant material and penetrated $\mathrm{AgNO}_{3}$. Subsequently, the penetration volume was calculated and recorded $\left(\mathrm{mm}^{3}\right)$ (Figure 1).

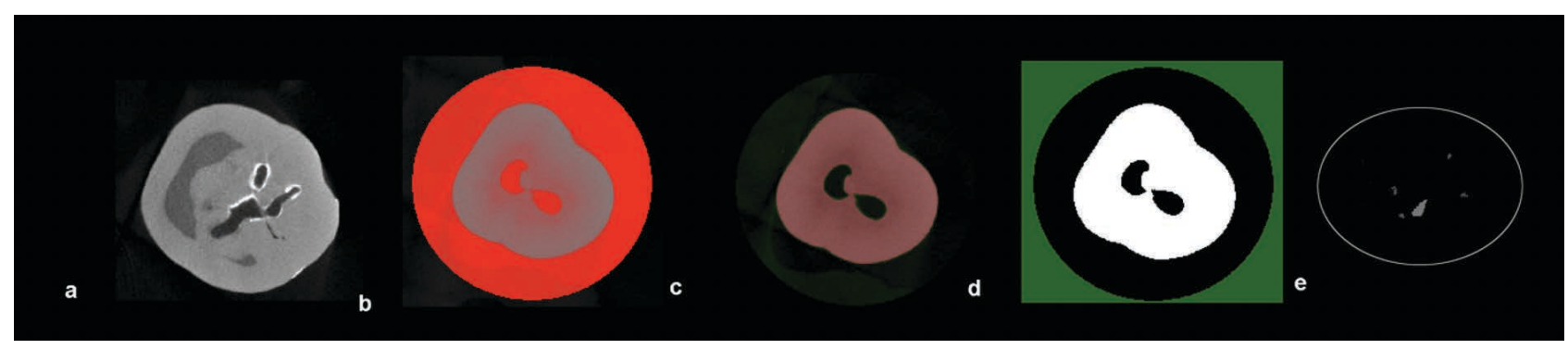

Figure 1. Micro CT images showing (a) axial image of the tooth with $\mathrm{AgNO}_{3}$, (b) VOI selection on images, (c,d) thresholding, (e), and binarization of the image by the gray-level histogram 


\section{Statistical Analysis}

SPSS 11.5 software was used in the analysis of the results. The mean $\pm \mathrm{SD}$ and median (minimum-maximum) were used for descriptive quantitative variables. In terms of quantitative variables, whether there is a statistical difference between the categories of qualitative variables with two categories was analyzed with Student's t-test since normal distribution assumptions were provided. In terms of quantitative variables, whether there is a statistical difference between the categories of qualitative variables with more than two categories was analyzed with One Way ANOVA test since normal distribution assumptions were provided. If there is a significant difference between these groups, the Tukey test was used in the comparison of the binary groups when the assumption of homogeneity of variances was provided, and the Games-Howell test was used when not provided. The statistical significance level was considered as $5 \%(\mathrm{p}<0.05)$.

\section{Results}

The mean volumetric marginal microleakage values of three different pit and fissure sealants with the noninvasive and invasive technique were given in Table 1 . The microleakage values of the invasive technique were found to be statistically significantly lower than the non-invasive technique for all sealants. In terms of the success of pit and fissure sealants, while Group 3 showed the minimum microleakage values, Group 1 showed the highest values. In statistical comparisons of two application techniques, a significant difference was observed between non-invasive and invasive techniques for Group 1, 2, and 3 ( $\mathrm{p}=0.001, \mathrm{p}<0.001$ and $\mathrm{p}<0.001$, respectively) (Table 1) (Figure 2 and 3).
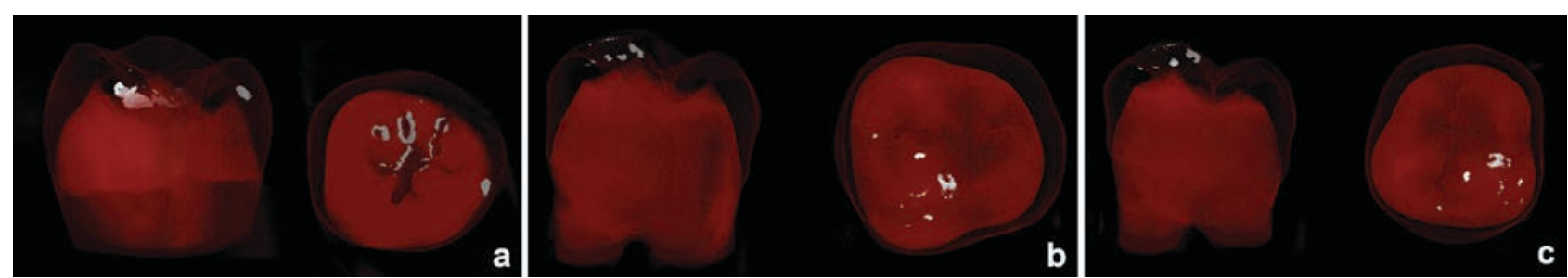

Figure 2. Micro-CT images of marginal microleakage of different pit and fissure sealant materials for non-invasive technique (White areas indicate leaking $\mathrm{AgNO}_{3}$ ) (a: Group 1, b: Group 2; c: Group 3).
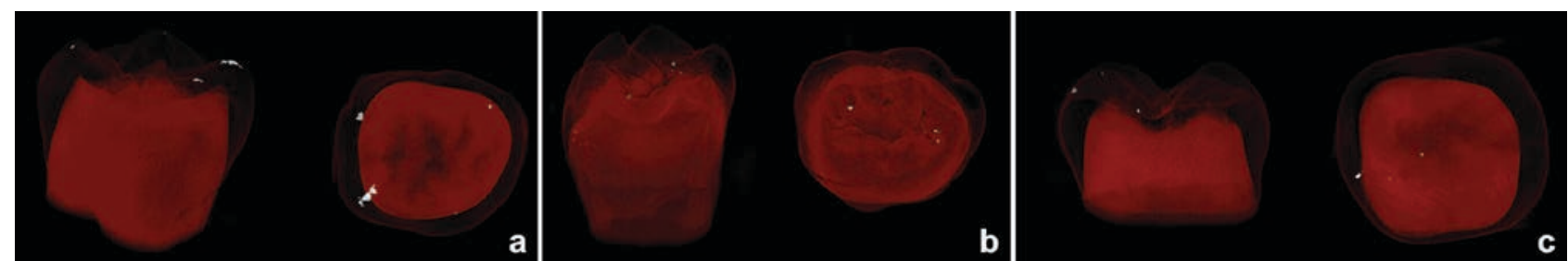

Figure 3. Micro-CT images of marginal microleakage of different pit and fissure sealant materials for invasive technique (White areas indicate leaking $\mathrm{AgNO}_{3}$ ) (a: Group 1, b: Group 2; c: Group 3)

Table 1. Statistical comparison of non-invasive and invasive techniques for all pit and fissure sealant materials regarding volumetric marginal microleakage values $\left(\mathrm{mm}^{3}\right)$

\begin{tabular}{|c|c|c|c|c|c|}
\hline \multirow{3}{*}{ Groups } & \multicolumn{4}{|c|}{ Application Technique } & \multirow{3}{*}{ p-value } \\
\hline & \multicolumn{2}{|c|}{ Non-Invasive } & \multicolumn{2}{|c|}{ Invasive } & \\
\hline & $\begin{array}{l}\operatorname{Mean} \pm \text { SD } \\
\left(\mathrm{mm}^{3}\right)\end{array}$ & $\begin{array}{c}\text { Median } \\
\text { (Min-Max) }\end{array}$ & $\begin{array}{c}\operatorname{Mean} \pm \mathbf{S D} \\
\left(\mathrm{mm}^{3}\right)\end{array}$ & $\begin{array}{c}\text { Median } \\
\text { (Min-Max) }\end{array}$ & \\
\hline Group 1 & $0.045 \pm 0.003$ & $\begin{array}{c}0.047 \\
(0.041-0.049)\end{array}$ & $0.035 \pm 0.003$ & $\begin{array}{c}0.037 \\
(0.031-0.039)\end{array}$ & $0.001 *$ \\
\hline Group 2 & $0.043 \pm 0.002$ & $\begin{array}{c}0.043 \\
(0.040-0.046)\end{array}$ & $0.016 \pm 0.002$ & $\begin{array}{c}0.018 \\
(0.011-0.019)\end{array}$ & $<0.001 *$ \\
\hline Group 3 & $0.036 \pm 0.005$ & $\begin{array}{c}0.033 \\
(0.032-0.043)\end{array}$ & $0,015 \pm 0,002$ & $\begin{array}{c}0.014 \\
(0.014-0.018)\end{array}$ & $<0.001 *$ \\
\hline
\end{tabular}

*indicates statistically significance for Student's t-test 
In a statistical comparison of volumetric marginal microleakage measurements of sealants, a significant difference was determined between the sealant materials in non-invasive technique $(\mathrm{p}=0.003)$ (Table 2). According to binary comparisons made for the determination of the groups that create a significant difference, only difference between Group 1-3 was found to be statistically significant $(\mathrm{p}=0.022)$ (Figure 2$)$. In invasive technique, a statistically significant difference was also found between the sealant materials $(\mathrm{p}<0.001)$ (Table 2$)$. According to the binary comparisons made for the determination of the groups that create a significant difference, the differences between Group 1-2 and Group 1-3 were significant $(\mathrm{p}<0.001$ and $\mathrm{p}<0.001$, respectively) (Figure 3 ).

Table 2. Statistical comparison of pit and fissure sealant materials with non-invasive and invasive techniques regarding marginal microleakage

\begin{tabular}{|c|c|c|c|c|c|c|c|}
\hline $\begin{array}{l}\text { Application } \\
\text { Technique }\end{array}$ & Groups & $\begin{array}{c}\text { Mean } \pm \text { SD } \\
\left(\mathrm{mm}^{3}\right)\end{array}$ & $\begin{array}{c}\text { Median } \\
\text { (Min-Max) }\end{array}$ & p-value & $\begin{array}{c}\text { Group 1-2 } \\
\text { p-value }\end{array}$ & $\begin{array}{c}\text { Group 1-3 } \\
\text { p-value }\end{array}$ & $\begin{array}{c}\text { Group 2-3 } \\
\text { p-value }\end{array}$ \\
\hline \multirow{3}{*}{ Non-Invasive } & Group 1 & $0.045 \pm 0.003$ & $\begin{array}{c}0.047 \\
(0.041-0.049)\end{array}$ & \multirow{3}{*}{$0.003 *$} & \multirow{3}{*}{0.434} & \multirow{3}{*}{$0.022 * *$} & \multirow{3}{*}{0.060} \\
\hline & Group 2 & $0.043 \pm 0.002$ & $\begin{array}{c}0.043 \\
(0.040-0.046)\end{array}$ & & & & \\
\hline & Group 3 & $0.036 \pm 0.005$ & $\begin{array}{c}0.033 \\
(0.032-0.043)\end{array}$ & & & & \\
\hline \multirow{3}{*}{ Invasive } & Group 1 & $0.035 \pm 0.003$ & $\begin{array}{c}0.037 \\
(0.031-0.039)\end{array}$ & \multirow{3}{*}{$<0.001 *$} & \multirow{3}{*}{$<0.001 * * *$} & \multirow{3}{*}{$<0.001 * * *$} & \multirow{3}{*}{0.862} \\
\hline & Group 2 & $0.016 \pm 0.002$ & $\begin{array}{c}0.018 \\
(0.011-0.019)\end{array}$ & & & & \\
\hline & Group 3 & $0.015 \pm 0.002$ & $\begin{array}{c}0.014 \\
(0.014-0.018)\end{array}$ & & & & \\
\hline
\end{tabular}

**indicates statistical significance for the One Way ANOVA test

***indicates statistical significance for the Games-Howell test

$* * * *$ indicates statistical significance for the Tukey test

\section{Discussion}

The pit and fissures on the tooth surfaces are susceptible areas for dental caries initiation ${ }^{17,18}$. Although various approaches are used in preventing occlusal caries, dental caries is still frequently encountered among oral health problems. Providing and maintaining oral hygiene with fluoride applications and the use of sealants are considered as the best preventive options ${ }^{17}$.

The adhesion of fissure sealants to enamel is quite associated with ensuring better marginal adaptation. The degree of marginal adaptation also affects the effectiveness, marginal sealing ability, and lifetime of fissure sealant material ${ }^{18,19}$. Besides, if applied material can not withstand microleakage, caries initiation or caries progression under the sealed surfaces occur, accordingly, treatment failure is observed in the tooth-material interface ${ }^{11,19}$. This creates a difficulty in caries diagnosis and treatment and hinders the success of fissure sealant application. ${ }^{11}$ Therefore, due to the critical importance of the sealing ability of fissure sealant materials in treatment success, this study was aimed to investigate the marginal microleakage of different pit and fissure sealants.
Resin-based fissure sealants have been used in preventive dentistry for many years ${ }^{20}$. Glass ionomer based materials can also be used as pit and fissure sealants due to better toleration of moisture/saliva contamination especially in partially erupted teeth and non-cooperative pediatric patients. Also, chemical bonding to dental hard tissues, the release of fluoride/acting as a fluoride reservoir, and easy handling properties are the main reasons for preferring glass ionomers ${ }^{21,22}$. Also, in the use of resin-containing materials, although the polymerization shrinkage leading to microleakage has been reduced with the improvement of adhesive systems, polymerization shrinkage is not seen in glass ionomers ${ }^{21,22}$. Giomer based fissure sealants are used in caries prevention in recent years $^{6}$. Based on this point, resin, high viscosity glass ionomer, and giomer based sealant materials were used in this study. Due to the in-vitro nature of our study, the inability to simulate all the physiological oral conditions is the limitation of this study. However, all samples were thermocycled to reflect the intraoral thermal changes ${ }^{19}$.

In dentistry, microleakage is one of the important criteria in the evaluation of the success of restorative materials. To date, the most commonly used method for 
microleakage evaluation is dye penetration test. However, although many studies evaluated the microleakage of the restorative materials or pit and fissure sealants using dye penetration test $\mathrm{t}^{2,6,10,19,22,23}$, Micro-CT method is the current and popular approach for performing microleakage evaluation $^{13}$. In the Micro-CT method, it is possible for the entire margins to be visible for evaluation without damaging the samples and there is no loss of information. This method allows the accurate evaluation of bonding levels of the tested sealants or restorative materials ${ }^{24}$. On the other hand, it has been reported since glass ionomer based materials have hydrophilic properties, they can absorb the dye material resulting in false-positive findings ${ }^{7}$. For these reasons, marginal microleakage assessments were performed using Micro-CT in this study. In the marginal microleakage evaluation of the samples, the volumetric measurement of the silver nitrate leaking from the enamelsealant interface was taken as the criterion.

In-pit and fissure sealant applications, the invasive technique eliminates the organic material content and plaque by widening and deepening of pit and fissures. Besides, this approach exposes more reactive enamel tissue and allows thicker sealant layer which is more wear-resistant ${ }^{7,25}$. Singla et al. ${ }^{7}$ stated that sealant material penetrated deep with the preparation of fissure surfaces, thereby increasing retention and adaptation. Vineet and Tandon $^{17}$ reported that if the invasive technique was applied in fissure sealant application, marginal integrity was strengthened. Therefore, the authors concluded that the non-invasive technique should be used only with materials that presented well adaptation ability. Similarly, Herle et $a l .{ }^{10}$ reported that plaque and debris were present in the depths of pit and fissures with non-invasive technique in SEM analysis and the authors emphasized that invasive technique offers the best flow and adaptation results. Therefore, both invasive and non-invasive techniques were included in this study for microleakage evaluation of different pit and fissure sealant materials. Moreover, in invasive techniques, different types of burs were used in the mechanical surface preparation of enamel ${ }^{26,27}$. Geiger et al. ${ }^{27}$ reported that the least microleakage values were observed in use of tapered fissure diamond burs. In the present study, the tooth surfaces were prepared using tapered fissure diamond burs with water cooling.

According to the results of this study, the volumetric measurement values of marginal microleakage in all fissure sealant materials were found to be lower in invasive technique compared to non-invasive technique. This finding coincides with the other previous studies ${ }^{7-10}$. Hatibovic-Kofman et al. ${ }^{9}$ reported that the best results for microleakage were obtained when surface preparation was performed and Bagherian et al. ${ }^{8}$ also reported that microleakage values decreased by fissurotomy procedure. Previous studies have indicated that the quality of material penetration, marginal integrity and tooth-material adaptation, which were closely related to microleakage, were increased by using invasive technique and surface preparation $7,10,17,27$. However, considering that the prepared enamel surface will be vulnerable to the risk of secondary caries and marginal microleakage in case where the sealant is partially or completely lost, we -as the authors- recommend the cases applied invasive fissure sealant should be followed-up regularly.

In the literature, not many studies have evaluated the marginal microleakage of fissure sealants in invasive and non-invasive techniques using Micro-CT; microleakage evaluations were mostly performed with dye penetration test $^{2,6,10,19,22,23}$. In some studies using dye penetration ${ }^{7,10,28}$ although the microleakage values of resin-based fissure sealants were less than glass ionomer based sealants, in our study, microleakage values of glass ionomer-based fissure sealant were found to be significantly lower compared to resin-based sealant for both application techniques ( $p=0.022$ for non-invasive technique, $p<0.001$ for invasive technique). However, Fracasso et al. ${ }^{23}$ reported that glass ionomers presented significantly better results compared to resin-based sealants in terms of microleakage assessment, and the authors stated that this finding was associated with the physicochemical properties of glass ionomer materials. Similarly, Chen et $a{ }^{24}$ reported in the micro-CT study that the use of high viscosity glass ionomers (ART sealant) as fissure sealants showed lower leakage values than resin-based sealants. On the other hand, the authors also stated that adding energy to high viscosity glass ionomers had no significant effects on marginal leakage. In this study, the lower leakage results of glass ionomer can be attributed to the assessment technique and the high viscosity properties of glass ionomer material used. Based on the mentioned findings of our study, considering the clinical advantages of glass ionomers, these materials can be used as pit and fissure sealant as stated in other studies ${ }^{10,21,23,28}$. Indeed, Borsatto et al. ${ }^{29}$ reported that glass ionomer sealants offered better results in marginal microleakage evaluation with saliva contamination.

Giomer is the material consisting of methacrylatebased composite and surface pre-reacted glass ionomer (S-PRG) fillers ${ }^{6,30,31}$. In this study, there was no significant difference between giomer based and glass ionomer based fissure sealant for both application techniques (non-invasive and invasive) in terms of marginal microleakage $(p=0.060$ for non-invasive technique, $\mathrm{p}=0.862$ for invasive technique). However, giomer based fissure sealant has been found to have significantly lower microleakage values than resin fissure sealant in invasive technique $(\mathrm{p}<0.001)$. In this respect, it is possible to say that fissure sealants with giomer content can also be used in routine clinical practice. Similarly, Ataol et $a l .{ }^{6}$ reported that giomer sealants can be used in pediatric patients with high caries risk and cases of isolation difficulties. 


\section{Conclusions}

Considering the advantages of glass ionomer and giomer materials in routine clinical practice, it was possible to concluded that both glass ionomer-based Fuji IX GP and giomer-based BeautiSealant materials could be effective as an alternative to resin-based pit and fissure sealants.

\section{References}

1. Fernandes KS, Chalakkal P, de Ataide IN, Pavaskar R, Fernandes PP, Soni H. A comparison between three different pit and fissure sealants with regard to marginal integrity. J Conserv Dent, 2012;15:146-150.

2. Babaji P, Vaid S, Deep S, Mishra S, Srivastava M, Manjooran T. In vitro evaluation of shear bond strength and microleakage of different pit and fissure sealants. J Int Soc Prev Community Dent, 2016;6:S111-115.

3. Fuks AB, Peretz B. Pediatric Endodontics: Past and Present Perspectives and Future Directions. In: Fuks AB, Peretz B, editors. Pediatric Endodontics: Current Concepts in Pulp Therapy for Primary and Young Permanent Teeth (1st ed.). Switzerland: Springer International Publishing, 2016. pp: 1-5.

4. Newbrun E. Topical fluorides in caries prevention and management: a North American perspective. J Dent Educ, 2001;65:1078-1083.

5. Ahovuo-Saloranta A, Forss H, Walsh T, Nordblad A, Mäkelä M, Worthington HV. Pit and fissure sealants for preventing dental decay in permanent teeth. Cochrane Database Syst Rev, 2017;7:CD001830.

6. Ataol E, Ertan A, Cehreli ZC. Sealing effectiveness of fissure sealants bonded with universal adhesive systems: influence of different etching modes. J Adh Sci Tech, 2017;31:1626-1634.

7. Singla A, Garg S, Jindal SK, Suma Sogi HP, Sharma D. In vitro evaluation of marginal leakage using invasive and noninvasive technique of light cure glass ionomer and flowable polyacid modified composite resin used as pit and fissure sealant. Indian J Dent Res, 2011;22:205-209.

8. Bagherian A, Ahmadkhani M, Sheikhfathollahi M, Bahramabadinejad R. Microbial microleakage assessment of a new hydrophilic fissure sealant: a laboratory study. Pediatr Dent, 2013;35:194-198.

9. Hatibovic-Kofman S, Wright GZ, Braverman I. Microleakage of sealants after conventional, bur, and airabrasion preparation of pits and fissures. Pediatr Dent, 1998;20:173-176.

10. Herle GP, Joseph T, Varma B, Jayanthi M. Comparative evaluation of glass lonomer and resin based fissure sealant using noninvasive and invasive techniques--a SEM and microleakage study. J Indian Soc Pedod Prev Dent, 2004;22:56-62

11. Youssef MN, Youssef FA, Souza-Zaroni WC, Turbino ML, Vieira MMF. Effect of enamel preparation method on in vitro marginal microleakage of a flowable composite used as pit and fissure sealant. Int J Paediatr Dent, 2006;16:342-347.
12. Chen X, Cuijpers VMJI, Fan MW, Frencken JE. Validation of micro-CT against the section method regarding the assessment of marginal leakage of sealants. Aust Dent J, 2012;57:196-199.

13. AlHabdan AA. Review of microleakage evaluation tools. J Int Oral Health, 2017;9:141-145.

14. Mollica F, De Santis R, Ambrosio L, Nicolais L, Prisco D, Rengo S. Mechanical and leakage behaviour of the dentin-adhesive interface. J Mater Sci Mater Med, 2004;15:485-492.

15. Krithikadatta J, Gopikrishna V, Datta M. CRIS Guidelines (Checklist for Reporting In-vitro Studies): A concept note on the need for standardized guidelines for improving quality and transparency in reporting in-vitro studies in experimental dental research. J Conserv Dent, 2014;17:301-304.

16. Feldkamp LA, Goldstein SA, Parfitt AM, Jesion G, Kleerekoper M. The direct examination of three dimensional bone architecture in vitro by computed tomography. J Bone Miner Res, 1989;4:3-11.

17. Vineet D, Tandon S. Comparative evaluation of marginal integrity of two new fissure sealants using invasive and non-invasive techniques: a SEM study. J Clin Pediatr Dent, 2000;24:291-297.

18. Gunjal S, Nagesh L, Raju HG. Comparative evaluation of marginal integrity of glass ionomer and resin based fissure sealants using invasive and non-invasive techniques: an in vitro study. Indian J Dent Res, 2012;23:320-325.

19. Pardi V, Sinhoreti MA, Pereira AC, Ambrosano GM, de Meneghim MC. In vitro evaluation of microleakage of different materials used as pit-and-fissure sealants. Braz Dent J, 2006;17:49-52.

20. Beun S, Bailly C, Devaux J, Leloup G. Physical, mechanical and rheological characterization of resinbased pit and fissure sealants compared to flowable resin composites. Dent Mater, 2012;28:349-359.

21. Ashwin R, Arathi R. Comparative evaluation for microleakage between Fuji-VII glass ionomer cement and light-cured unfilled resin: a combined in vivo in vitro study. J Indian Soc Pedod Prev Dent, 2007;25:86-87.

22. Peng Y, Stark PC, Rich Jr A, Loo CY. Marginal microleakage of triage sealant under different moisture contamination. Pediatr Dent, 2011;33:203-206.

23. Fracasso MLC, Rios D, Machado MAAM, da Silva SMB, Abdo RCC. Evaluation of marginal microleakage and depth of penetration of glass ionomer cements used as occlusal sealants. J Appl Oral Sci, 2005;13:269-274.

24. Chen X, Cuijpers V, Fan M, Frencken JE. Marginal leakage of two newer glass-ionomer based sealant materials assessed using micro-CT. J Dent, 2010;38:731-735.

25. do Rego MA, de Araujo MA. Microleakage evaluation of pit and fissure sealants done with different procedures, materials, and laser after invasive technique. J Clin Pediatr Dent, 1999;24:63-68.

26. Garcia-Godoy F, de Araujo FB. Enhancement of fissure sealant penetration and adaptation: the enameloplasty technique. J Clin Pediatr Dent, 1994;19:13-18.

27. Geiger SB, Gulayev S, Weiss EI. Improving fissure sealant quality: Mechanical preparation and filling level. J Dent, 2000;28:407-412. 
28. Singh SH, Pathak A. Comparison of Microleakage of Glass Ionomer Sealant in Etched and Non Etched Fissure Enamel with Resin Based Sealant- An In Vitro Study. Int J Oral Health Med Res, 2016;2:51-53.

29. Borsatto MC, Corona SA, Alves AG, Chimello DT, Catirse $\mathrm{AB}$, Palma-Dibb RG. Influence of salivary contamination on marginal microleakage of pit and fissure sealants. Am J Dent, 2004;17:365-367.

30. Gordan VV, Blaser PK, Watson RE, Mjör IA, McEdward DL, Sensi LG, et al. A clinical evaluation of a giomer restorative system containing surface prereacted glass ionomer filler: results from a 13-year recall examination. $\mathrm{J}$ Am Dent Assoc, 2014;145:1036-1043.

31. Kaga M, Kakuda S, Ida Y, Toshima H, Hashimoto M, Endo K, Sano H. Inhibition of enamel demineralization by buffering effect of S-PRG filler-containing dental sealant. Eur J Oral Sci, 2014;122:78-83.
Conflict of Interests: Nothing to declare.

Financial Disclosure Statement: Nothing to declare.

Human Rights Statement: All the procedures on humans were conducted in accordance with the Helsinki Declaration of 1975, as revised 2000. Consent was obtained from the patient/s and approved for the current study by national ethical committee.

Animal Rights Statement: None required.

Received on February 4, 2021.

Revised on April 2, 2021.

Accepted on May 29, 2021.

Correspondence

Akif Demirel Department of Pediatric Dentistry Faculty of Dentistry, Ankara University Ankara, Turkey

e-mail: akifdemirel@ankara.edu.tr 